Network Working Group

Request for Comments: 901

Obsoletes: RFCs 880,840
J. Reynolds

J. Postel

ISI

June 1984

OFFICIAL ARPA-INTERNET PROTOCOLS

Status of this Memo

This memo is an official status report on the protocols used in the ARPA-Internet community.

Introduction

This RFC identifies the documents specifying the official protocols used in the Internet. Annotations identify any revisions or changes planned.

To first order, the official protocols are those in the "Internet Protocol Transition Workbook" (IPTW) dated March 1982. There are several protocols in use that are not in the IPTW. A few of the protocols in the IPTW have been revised. Notably, the mail protocols have been revised and issued as a volume titled "Internet Mail Protocols" dated November 1982. Telnet and the most useful option protocols were issued by the NIC in a booklet entitled "Internet Telnet Protocol and Options" (ITP), dated June 1983. Some protocols have not been revised for many years, these are found in the old "ARPANET Protocol Handbook" (APH) dated January 1978. There is also a volume of protocol related information called the "Internet Protocol Implementers Guide" (IPIG) dated August 1982.

This document is organized as a sketchy outline. The entries are protocols (e.g., Transmission Control Protocol). In each entry there are notes on status, specification, comments, other references, dependencies, and contact.

The status is one of: required, recommended, elective, or experimental.

The specification identifies the protocol defining documents.

The comments describe any differences from the specification or problems with the protocol.

The other references identify documents that comment on or expand on the protocol.

The dependencies indicate what other protocols are called upon by this protocol. 
The contact indicates a person who can answer questions about the protocol.

In particular, the status may be:

required

- all hosts must implement the required protocol,

recommended

- all hosts are encouraged to implement the recommended protocol,

elective

- hosts may implement or not the elective protocol,

experimental

- hosts should not implement the experimental protocol unless they are participating in the experiment and have coordinated their use of this protocol with the contact person, and

none

- this is not a protocol.

For further information about protocols in general, please contact:

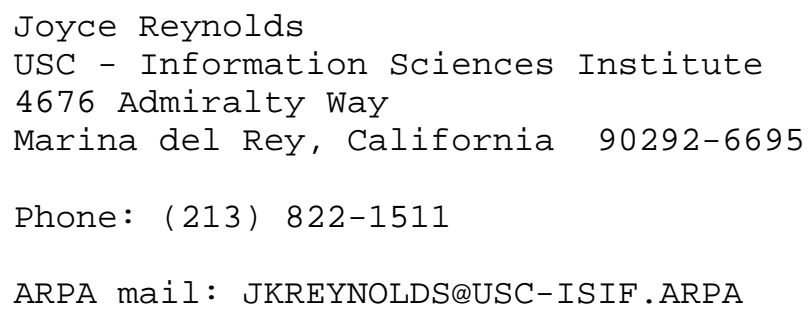


Overview

Catenet Model

STATUS: None

SPECIFICATION: IEN 48 (in IPTW)

COMMENTS :

Gives an overview of the organization and principles of the Internet.

Could be revised and expanded.

OTHER REFERENCES :

RFC 871 - A Perspective on the ARPANET Reference Model

DEPENDENCIES :

CONTACT: Postel@USC-ISIF.ARPA

Network Level

Internet Protocol (IP)

STATUS: Required

SPECIFICATION: RFC 791 (in IPTW)

\section{COMMENTS :}

This is the universal protocol of the Internet. This datagram protocol provides the universal addressing of hosts in the Internet.

A few minor problems have been noted in this document.

The most serious is a bit of confusion in the route options. The route options have a pointer that indicates which octet of the route is the next to be used. The confusion is between the phrases "the pointer is relative to this option" and "the smallest legal value for the pointer is 4". If you are confused, forget about the relative part, the pointer begins at 4 .

Another important point is the alternate reassembly procedure suggested in RFC 815. 


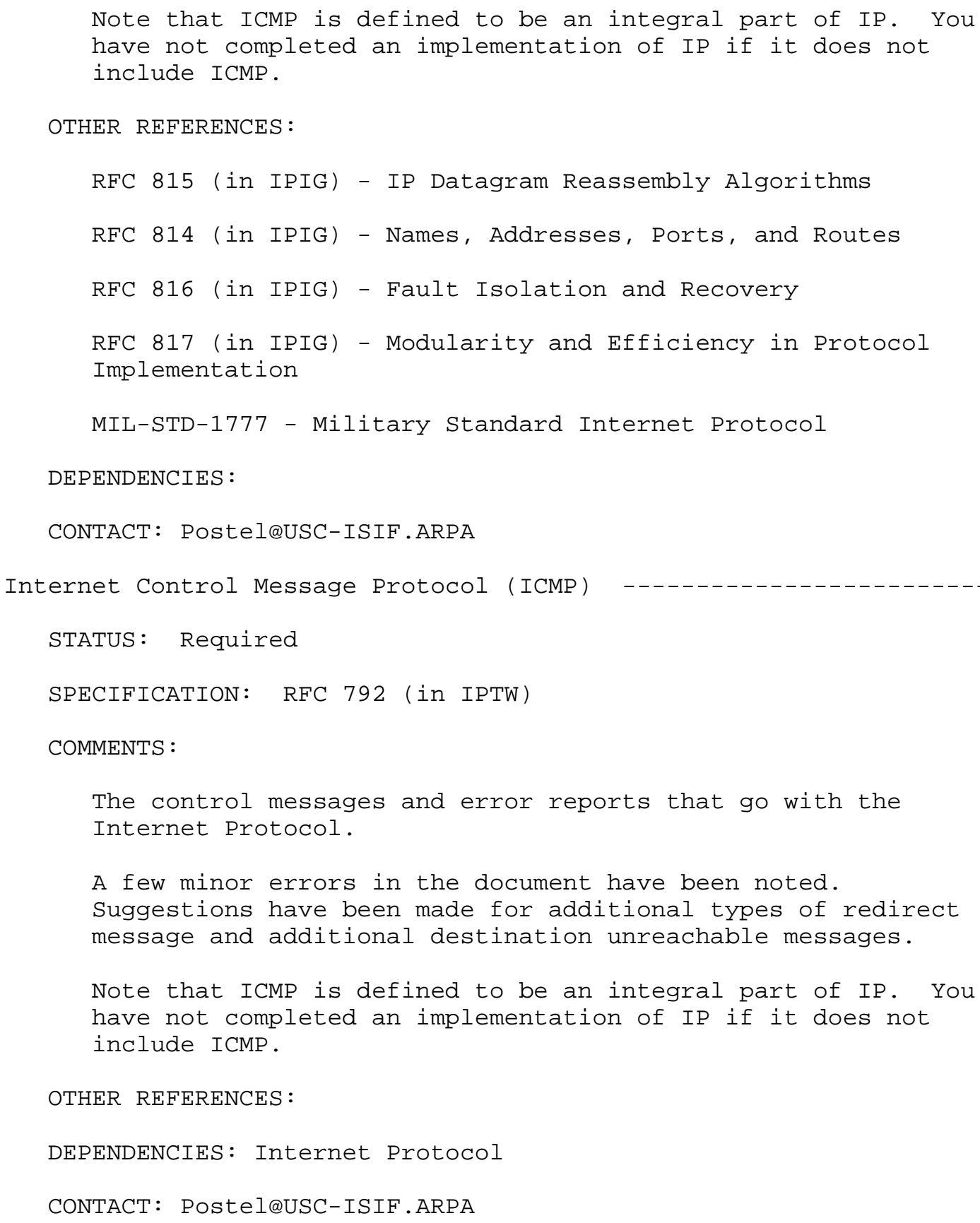


Host Level

User Datagram Protocol (UDP)

STATUS: Recommended

SPECIFICATION: RFC 768 (in IPTW)

COMMENTS :

Provides a datagram service to applications. Adds port addressing to the IP services.

The only change noted for the UDP specification is a minor clarification that if in computing the checksum a padding octet is used for the computation it is not transmitted or counted in the length.

OTHER REFERENCES :

DEPENDENCIES: Internet Protocol

CONTACT: Postel@USC-ISIF.ARPA

Transmission Control Protocol (TCP)

STATUS: Recommended

SPECIFICATION: RFC 793 (in IPTW)

COMMENTS :

Provides reliable end-to-end data stream service.

Many comments and corrections have been received for the TCP specification document. These are primarily document bugs rather than protocol bugs.

Event Processing Section: There are many minor corrections and clarifications needed in this section.

Push: There are still some phrases in the document that give a "record mark" flavor to the push. These should be further clarified. The push is not a record mark.

Listening Servers: Several comments have been received on difficulties with contacting listening servers. There should be some discussion of implementation issues for servers, and 
some notes on alternative models of system and process organization for servers.

Maximum Segment Size: The maximum segment size option should be generalized and clarified. It can be used to either increase or decrease the maximum segment size from the default. The TCP Maximum Segment Size is the IP Maximum Datagram Size minus forty. The default IP Maximum Datagram Size if 576. The default TCP Maximum Segement Size is 536. For further discussion, see RFC 879.

Idle Connections: There have been questions about automatically closing idle connections. Idle connections are ok, and should not be closed. There are several cases where idle connections arise, for example, in Telnet when a user is thinking for a long time following a message from the server computer before his next input. There is no TCP "probe" mechanism, and none is needed.

Queued Receive Data on Closing: There are several points where it is not clear from the description what to do about data received by the TCP but not yet passed to the user, particularly when the connection is being closed. In general, the data is to be kept to give to the user if he does a RECV call.

Out of Order segments: The description says that segments that arrive out of order, that is, are not exactly the next segment to be processed, may be kept on hand. It should also point out that there is a very large performance penalty for not doing so.

User Time Out: This is the time out started on an open or send call. If this user time out occurs the user should be notified, but the connection should not be closed or the TCB deleted. The user should explicitly ABORT the connection if he wants to give up.

OTHER REFERENCES:

RFC 813 (in IPIG) - Window and Acknowledgement strategy in TCP

RFC 814 (in IPIG) - Names, Addresses, Ports, and Routes

RFC 816 (in IPIG) - Fault Isolation and Recovery

RFC 817 (in IPIG) - Modularity and Efficiency in Protocol

Implementation 


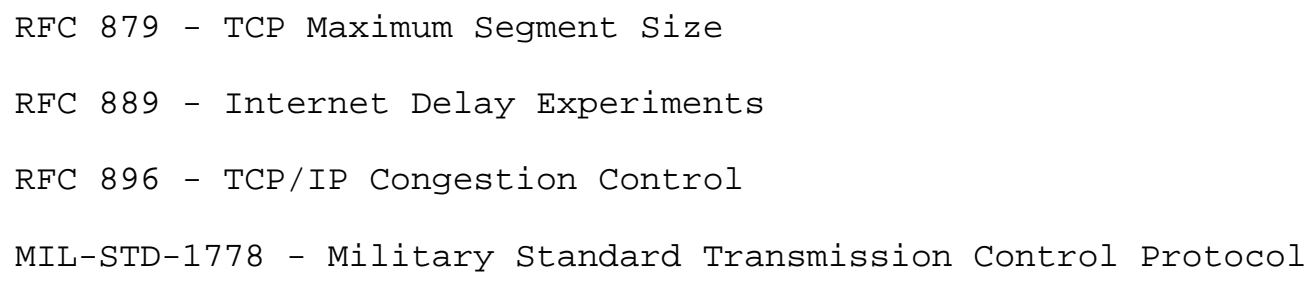




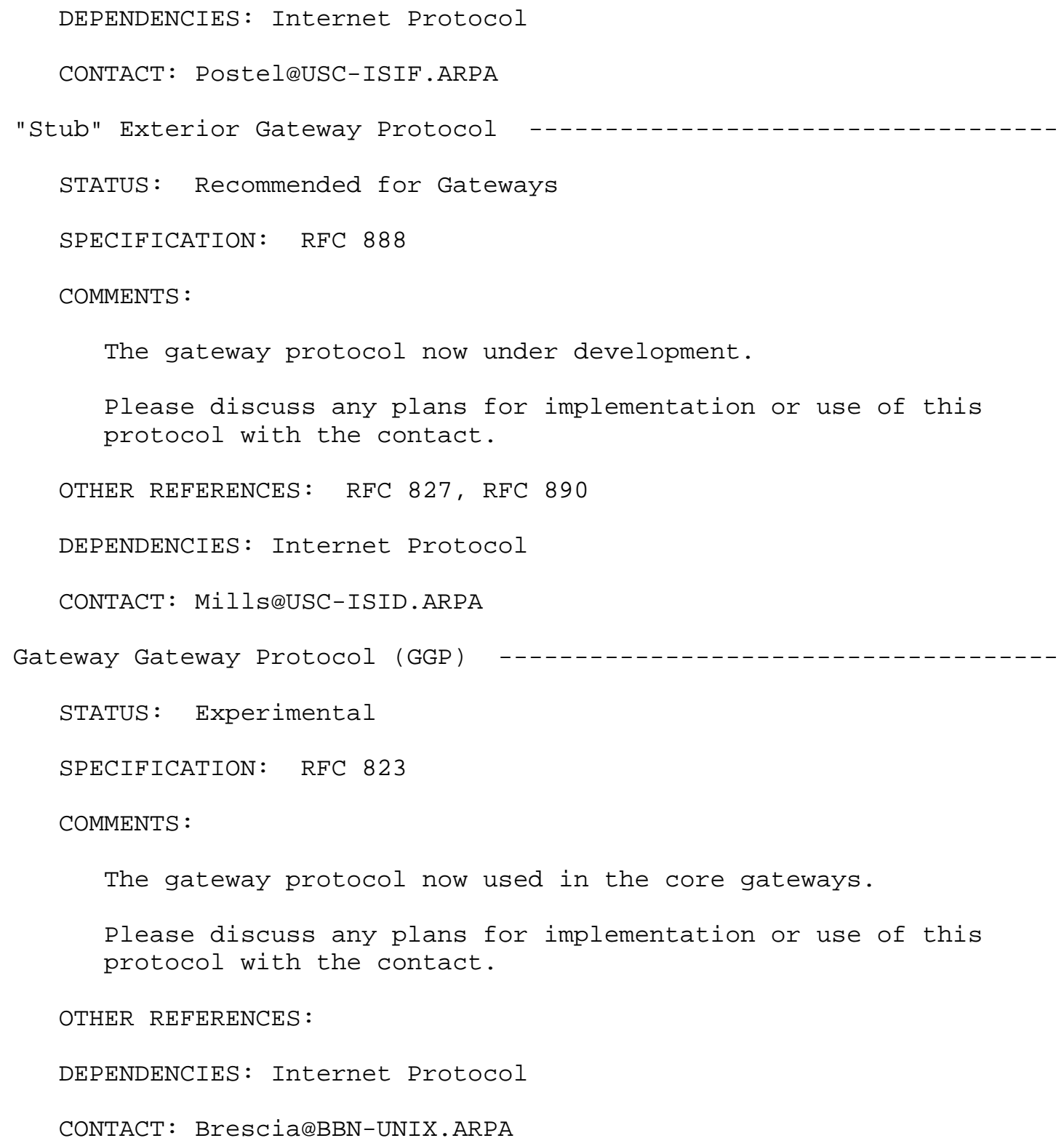




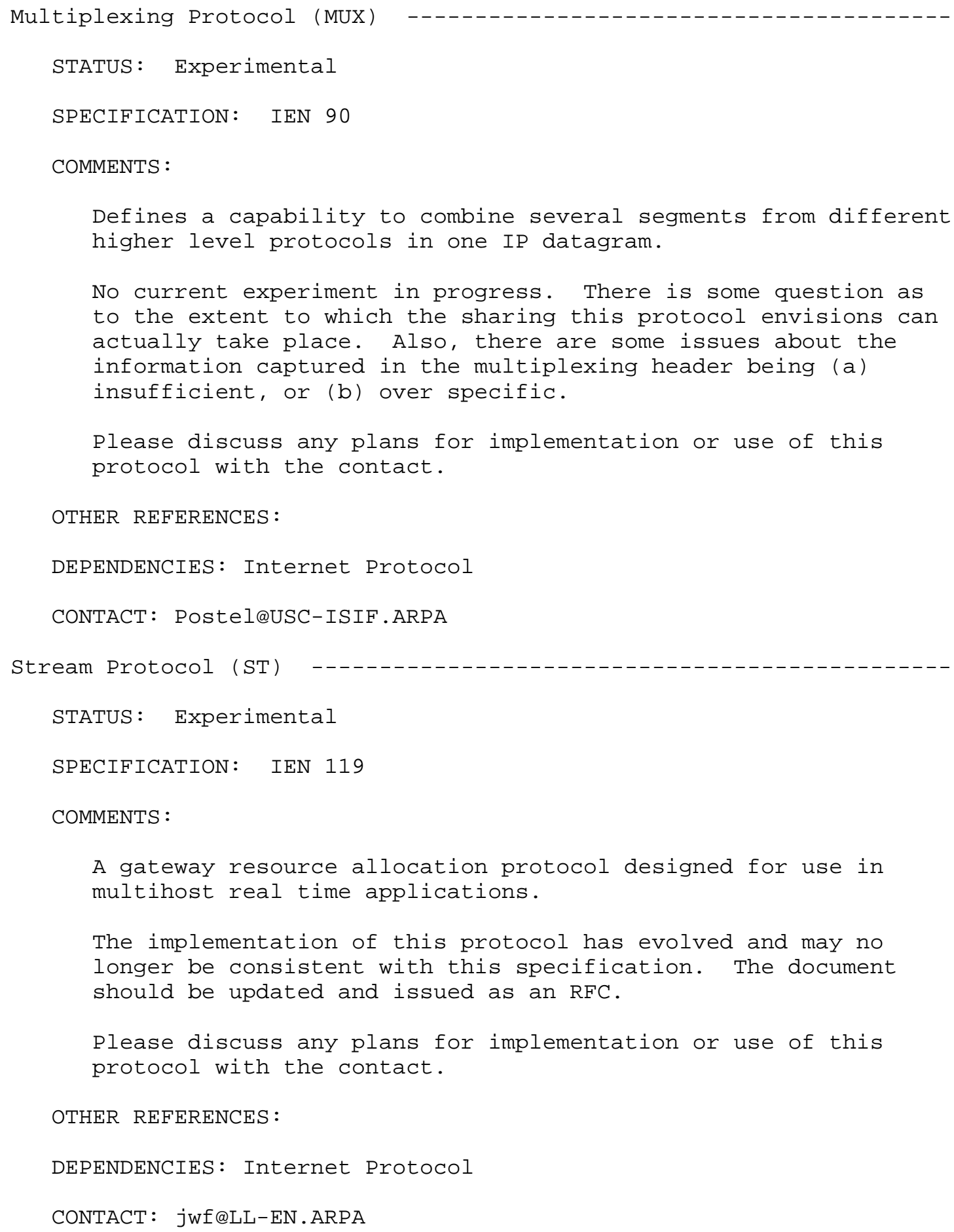




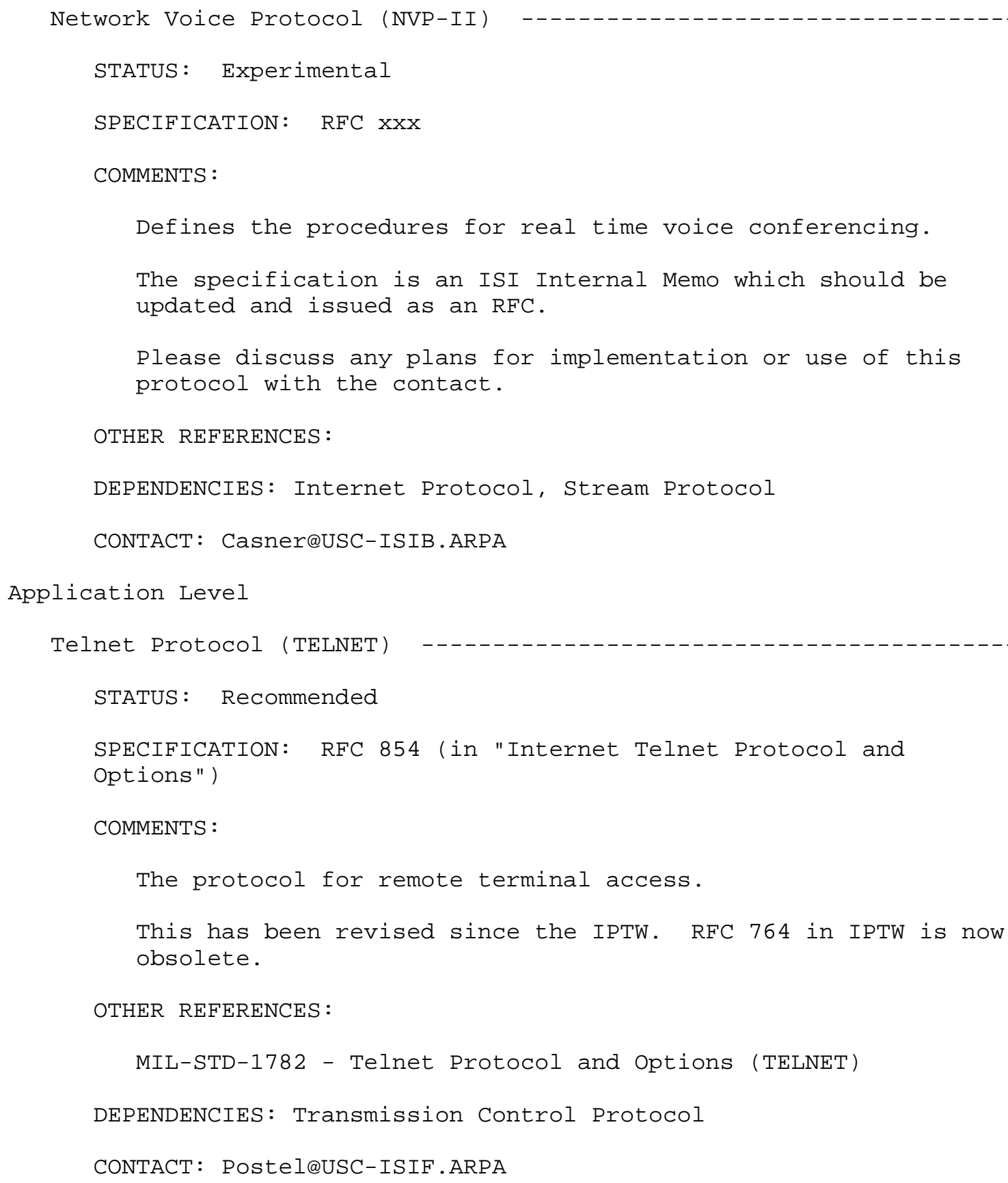


Telnet Options (TELNET-OPTIONS)

\section{STATUS: Elective}

SPECIFICATION: General description of options: RFC 855

(in "Internet Telnet Protocol and Options")

$\begin{array}{cl}\text { Number } & \text { Name } \\ 0 & \text { Binary Transmission } \\ 1 & \text { Echo } \\ 2 & \text { Reconnection } \\ 3 & \text { Suppress Go Ahead } \\ 4 & \text { Approx Message Size Negotiation } \\ 5 & \text { Status } \\ 6 & \text { Timing Mark } \\ 7 & \text { Remote Controlled Trans and Echo } \\ 8 & \text { Output Line Width } \\ 9 & \text { Output Page Size } \\ 10 & \text { Output Carriage-Return Disposition } \\ 11 & \text { Output Horizontal Tabstops } \\ 12 & \text { Output Horizontal Tab Disposition } \\ 13 & \text { Output Formfeed Disposition } \\ 14 & \text { Output Vertical Tabstops } \\ 15 & \text { Output Vertical Tab Disposition } \\ 16 & \text { Output Linefeed Disposition } \\ 17 & \text { Extended ASCII } \\ 18 & \text { Logout } \\ 19 & \text { Byte Macro } \\ 20 & \text { Data Entry Terminal } \\ 21 & \text { SUPDUP } \\ 22 & \text { SUPDUP Output } \\ 23 & \text { Send Location } \\ 25 & \text { Terminal Type } \\ 255 & \text { Extended-Options-List } \\ & \end{array}$

\begin{tabular}{|c|c|c|c|c|}
\hline $\mathrm{FC}$ & NIC & ITP & $\mathrm{APH}$ & USE \\
\hline 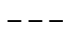 & ----- & --- & --- & --- \\
\hline 856 & ----- & yes & obs & yes \\
\hline 57 & ----- & yes & obs & yes \\
\hline & 15391 & no & yes & no \\
\hline 58 & ----- & yes & obs & yes \\
\hline & 15393 & no & yes & no \\
\hline 859 & ----- & yes & obs & yes \\
\hline 60 & ----- & yes & obs & yes \\
\hline 726 & 39237 & no & yes & no \\
\hline . & 20196 & no & yes & no \\
\hline & 20197 & no & yes & no \\
\hline 652 & 31155 & no & yes & no \\
\hline 653 & 31156 & no & yes & no \\
\hline 654 & 31157 & no & yes & no \\
\hline 655 & 31158 & no & yes & no \\
\hline 656 & 31159 & no & yes & no \\
\hline 657 & 31160 & no & yes & no \\
\hline 658 & 31161 & no & yes & no \\
\hline 698 & 32964 & no & yes & no \\
\hline 727 & 40025 & no & yes & no \\
\hline 735 & 42083 & no & yes & no \\
\hline 732 & 41762 & no & yes & no \\
\hline 736 & 42213 & no & yes & no \\
\hline 749 & 45449 & no & no & no \\
\hline 779 & ----- & no & no & no \\
\hline 884 & ----- & no & no & yes \\
\hline 885 & ----- & no & no & yes \\
\hline 861 & ----- & yes & obs & yes \\
\hline
\end{tabular}

The ITP column indicates if the specification is included in the Internet Telnet Protocol and Options. The APH column indicates if the specification is included in the ARPANET Protocol Handbook. The USE column of the table above indicates which options are in general use.

COMMENTS :

The Binary Transmission, Echo, Suppress Go Ahead, Status, Timing Mark, and Extended Options List options have been 
recently updated and reissued. These are the most frequently implemented options.

The remaining options should be reviewed and the useful ones should be revised and reissued. The others should be eliminated.

The following are recommended: Binary Transmission, Echo, Suppress Go Ahead, Status, Timing Mark, and Extended Options List.

OTHER REFERENCES:

DEPENDENCIES: Telnet

CONTACT: Postel@USC-ISIF.ARPA

File Transfer Protocol (FTP)

STATUS: Recommended

SPECIFICATION: RFC 765 (in IPTW)

\section{COMMENTS:}

The protocol for moving files between Internet hosts. Provides for access control and negotiation of file parameters.

There are a number of minor corrections to be made. A major change is the deletion of the mail commands, and a major clarification is needed in the discussion of the management of the data connection. Also, a suggestion has been made to include some directory manipulation commands (RFC 775).

Even though the MAIL features are defined in this document, they are not to be used. The SMTP protocol is to be used for all mail service in the Internet.

Data Connection Management:

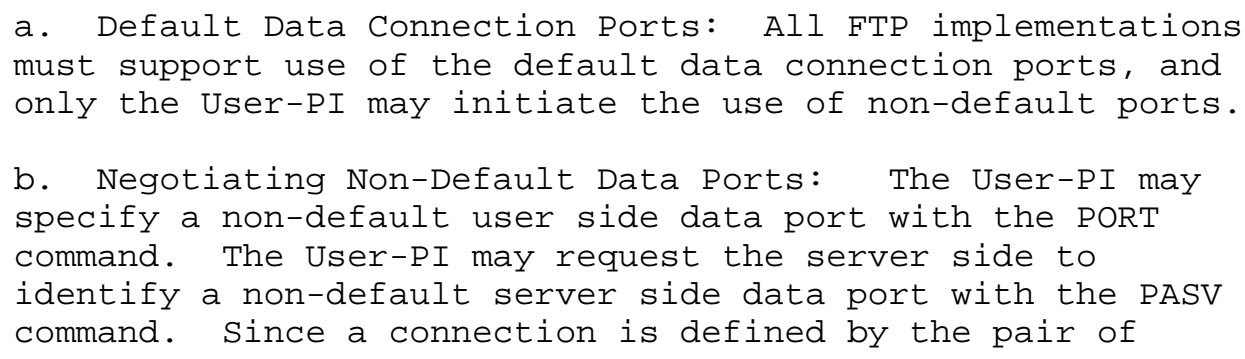


addresses, either of these actions is enough to get a different data connection, still it is permitted to do both commands to use new ports on both ends of the data connection.

c. Reuse of the Data Connection: When using the stream mode of data transfer the end of the file must be indicated by closing the connection. This causes a problem if multiple files are to be transfered in the session, due to need for TCP to hold the connection record for a time out period to guarantee the reliable communication. Thus the connection can not be reopened at once.

There are two solutions to this problem. The first is to negotiate a non-default port (as in (b) above). The second is to use another transfer mode.

A comment on transfer modes. The stream transfer mode is inherently unreliable, since one can not determine if the connection closed prematurely or not. The other transfer modes (Block, Compressed) do not close the connection to indicate the end of file. They have enough FTP encoding that the data connection can be parsed to determine the end of the file. Thus using these modes one can leave the data connection open for multiple file transfers.

Why this was not a problem with the old NCP FTP:

The NCP was designed with only the ARPANET in mind. The ARPANET provides very reliable service, and the NCP counted on it. If any packet of data from an NCP connection were lost or damaged by the network the NCP could not recover. It is a tribute to the ARPANET designers that the NCP FTP worked so well.

The TCP is designed to provide reliable connections over many different types of networks and interconnections of networks. TCP must cope with a set of networks that can not promise to work as well as the ARPANET. TCP must make its own provisions for end-to-end recovery from lost or damaged packets. This leads to the need for the connection phase-down time-out. The NCP never had to deal with acknowledgements or retransmissions or many other things the TCP must do to make connection reliable in a more complex world.

LIST and NLST: 
There is some confusion about the LIST an NLST commands, and what is appropriate to return. Some clarification and motivation for these commands should be added to the specification.

OTHER REFERENCES :

RFC 678 - Document File Format Standards

MIL-STD-1780 - File Transfer Protocol (FTP)

DEPENDENCIES: Transmission Control Protocol

CONTACT: Postel@USC-ISIF.ARPA

Trivial File Transfer Protocol (TFTP)

STATUS: Elective

SPECIFICATION: RFC 783 (in IPTW)

COMMENTS :

A very simple file moving protocol, no access control is provided.

No known problems with this specification. This is in use in several local networks.

OTHER REFERENCES :

DEPENDENCIES: User Datagram Protocol

CONTACT: Postel@USC-ISIF.ARPA

Simple Mail Transfer Protocol (SMTP)

STATUS: Recommended

SPECIFICATION: RFC 821 (in "Internet Mail Protocols")

COMMENTS :

The procedure for transmitting computer mail between hosts.

This has been revised since the IPTW, it is in the "Internet Mail Protocols" volume of November 1982. RFC 788 (in IPTW) is obsolete. 
There have been many misunderstandings and errors in the early implementations. Some documentation of these problems can be found in the file [ISIF] $<$ SMTP $>$ MAIL.ERRORS.

Some minor differences between RFC 821 and RFC 822 should be resolved.

OTHER REFERENCES:

RFC 822 - Mail Header Format Standards

This has been revised since the IPTW, it is in the "Internet Mail Protocols" volume of November 1982. RFC 733 (in IPTW) is obsolete. Further revision of RFC 822 is needed to correct some minor errors in the details of the specification.

MIL-STD-1781 - Simple Mail Transfer Protocol (SMTP)

DEPENDENCIES: Transmission Control Protocol

CONTACT: Postel@USC-ISIF.ARPA

Resource Location Protocol (RLP)

STATUS: Elective

SPECIFICATION: $\quad$ RFC 887

COMMENTS :

A resource location protocol for use in the ARPA-Internet. This protocol utilizes the User Datagram Protocol (UDP) which in turn calls on the Internet Protocol to deliver its datagrams.

OTHER REFERENCES :

DEPENDENCIES: User Datagram Protocol

CONTACT: Accetta@CMU-CS-A.ARPA 


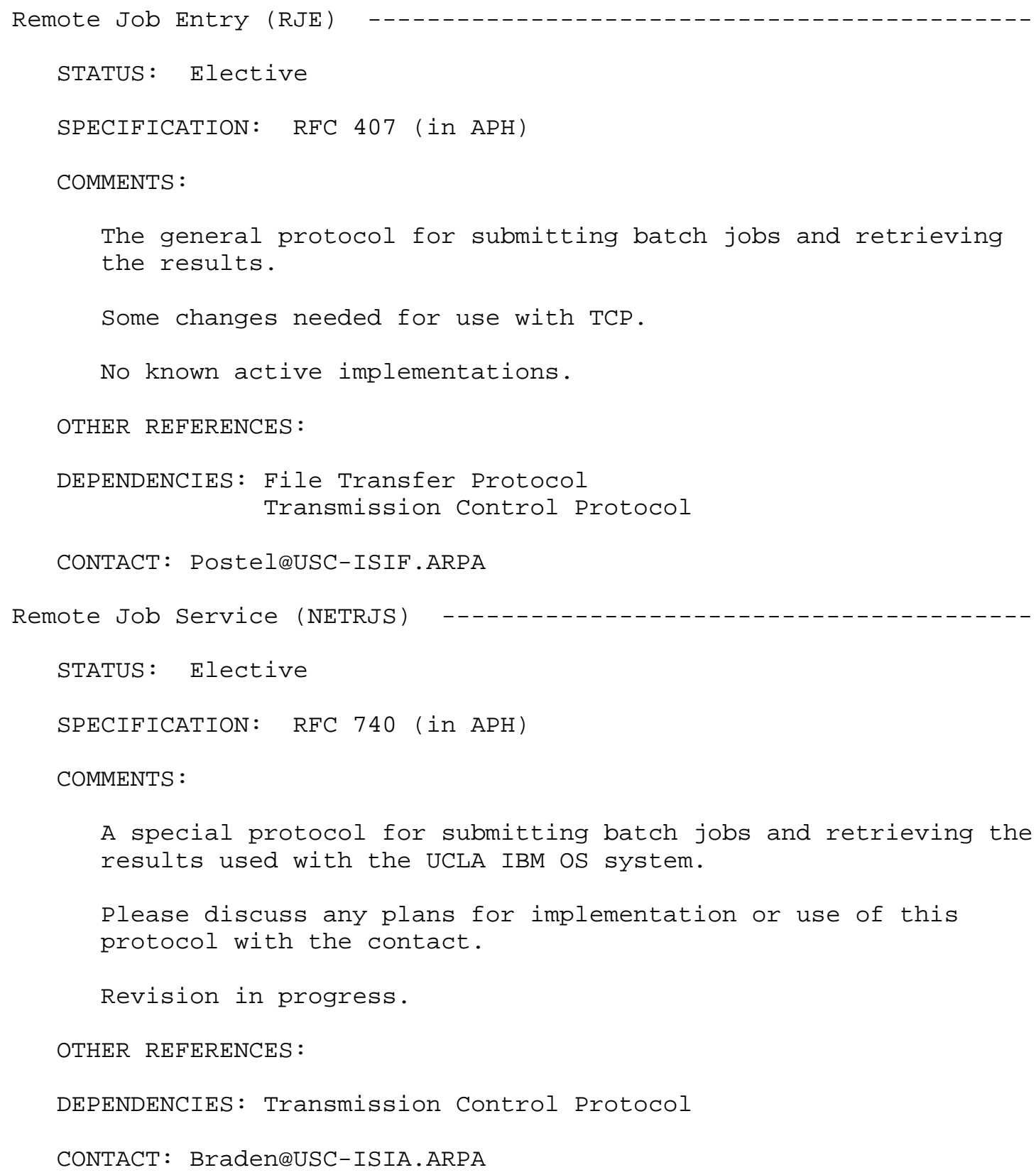




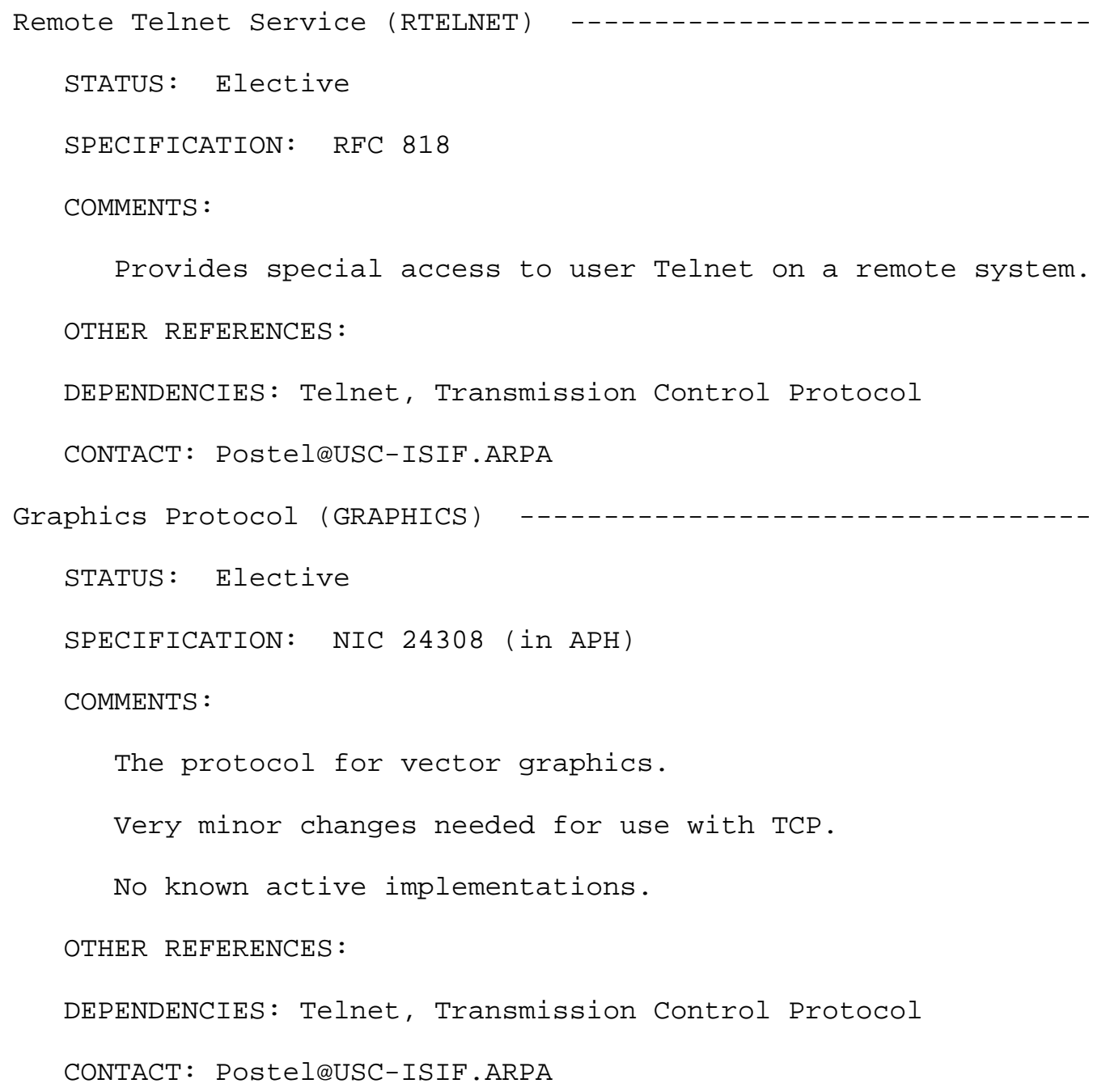




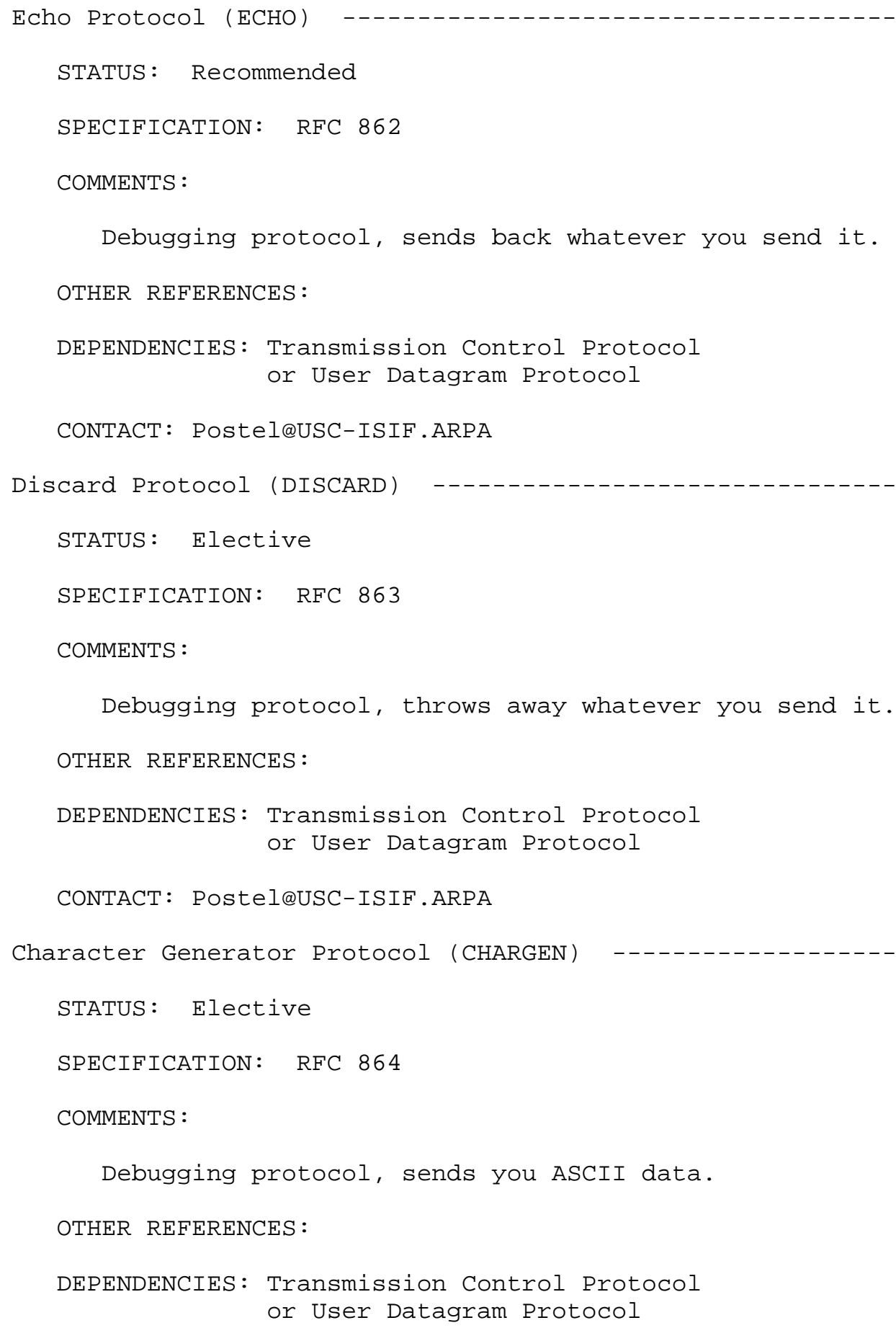




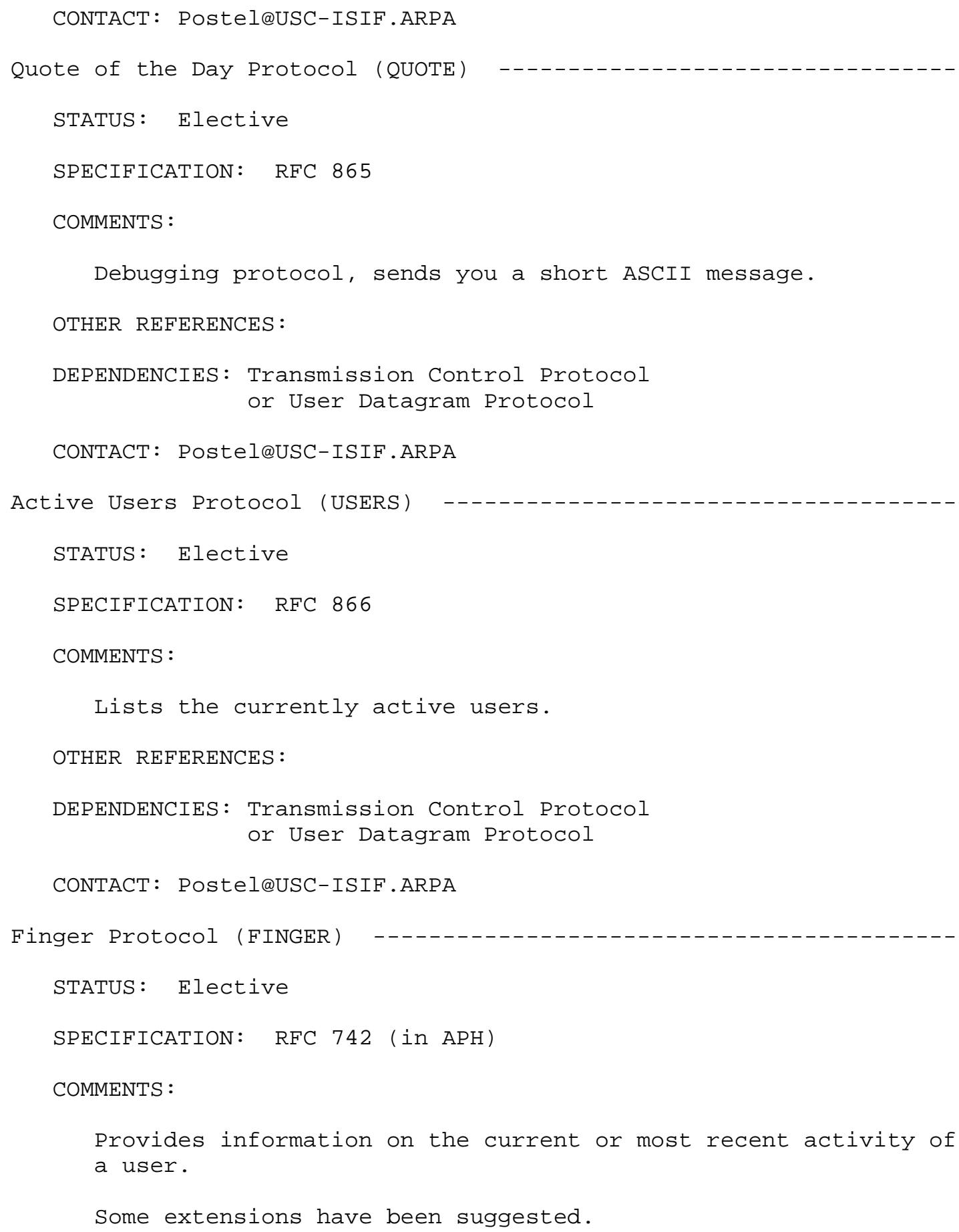




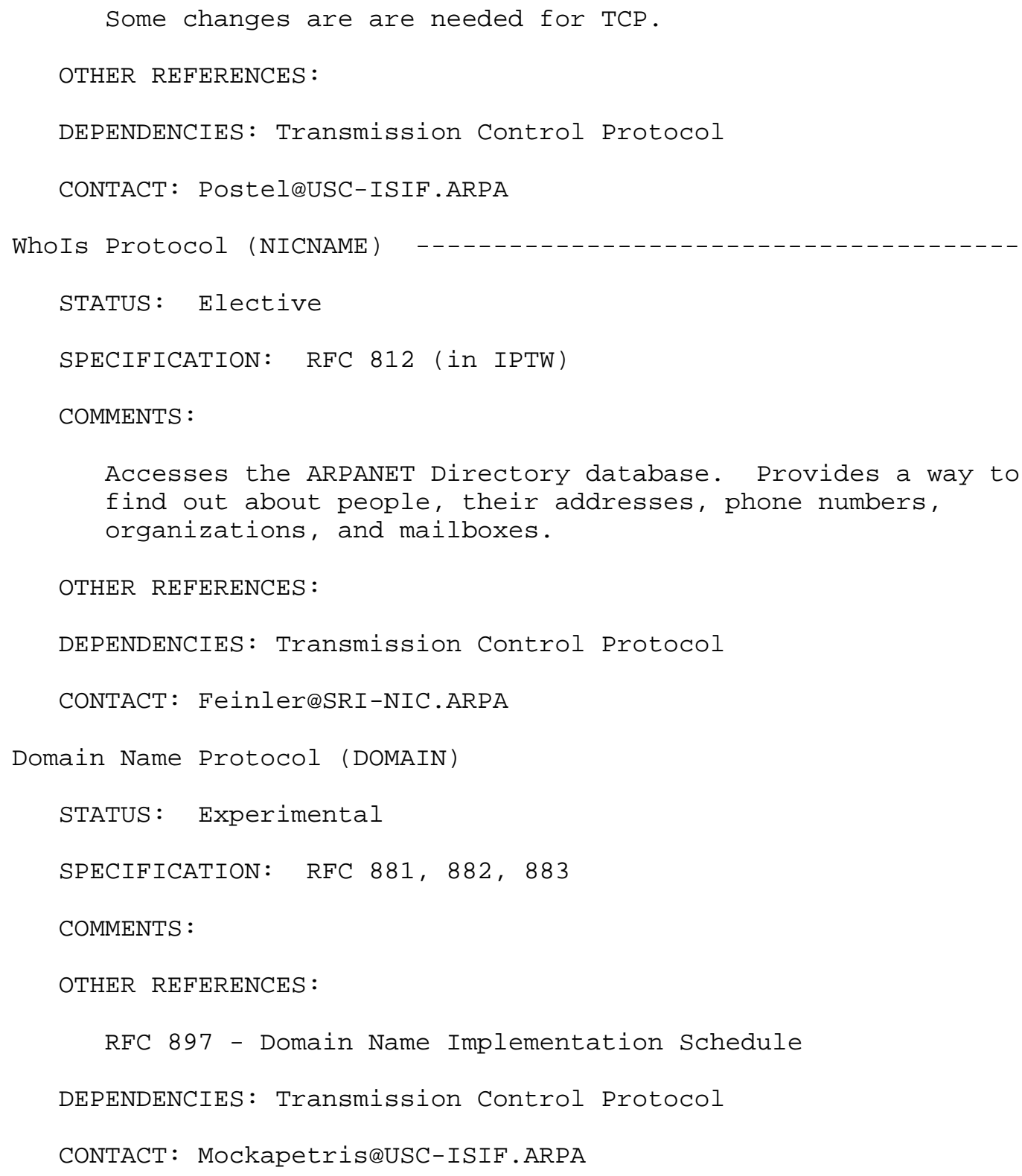




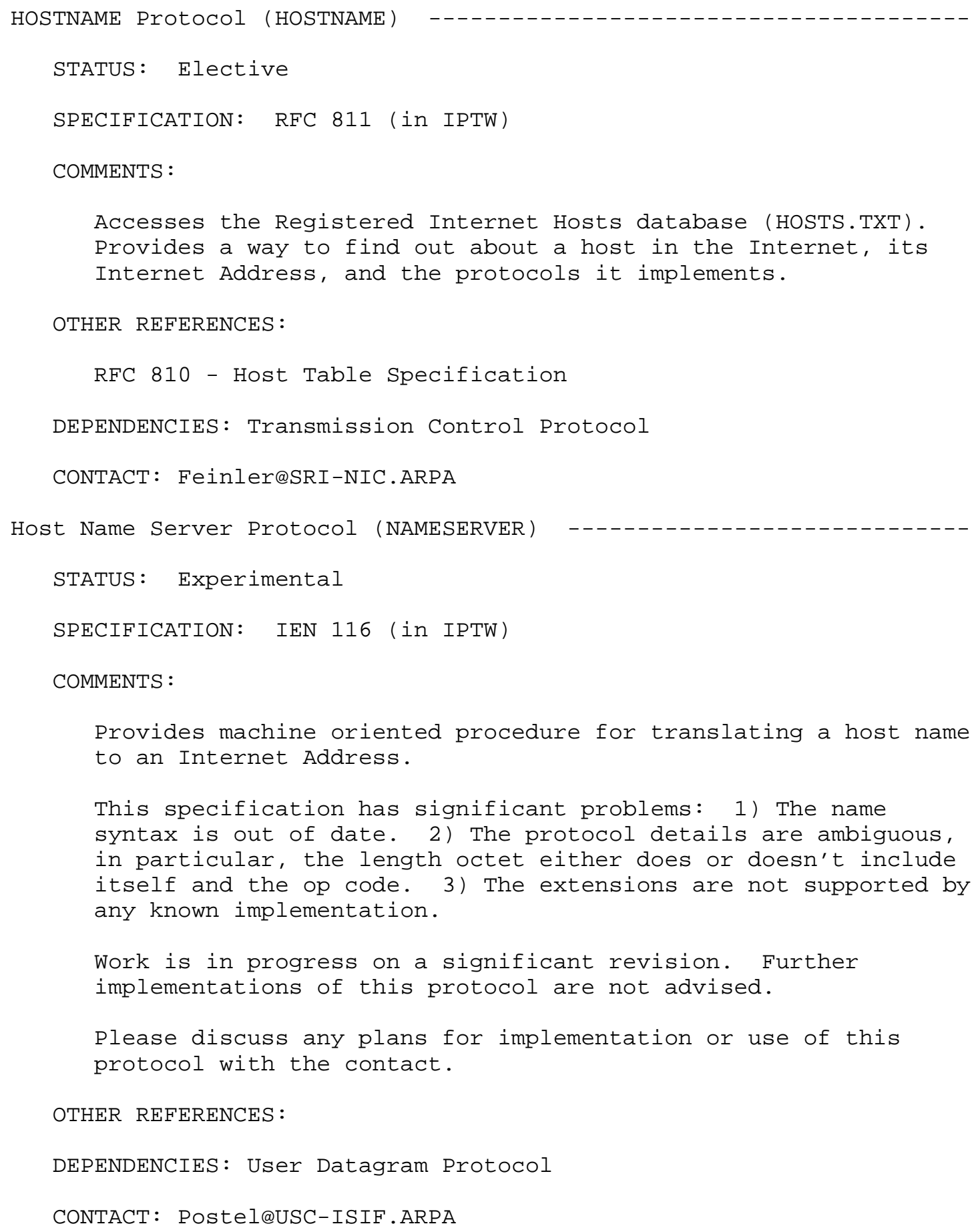




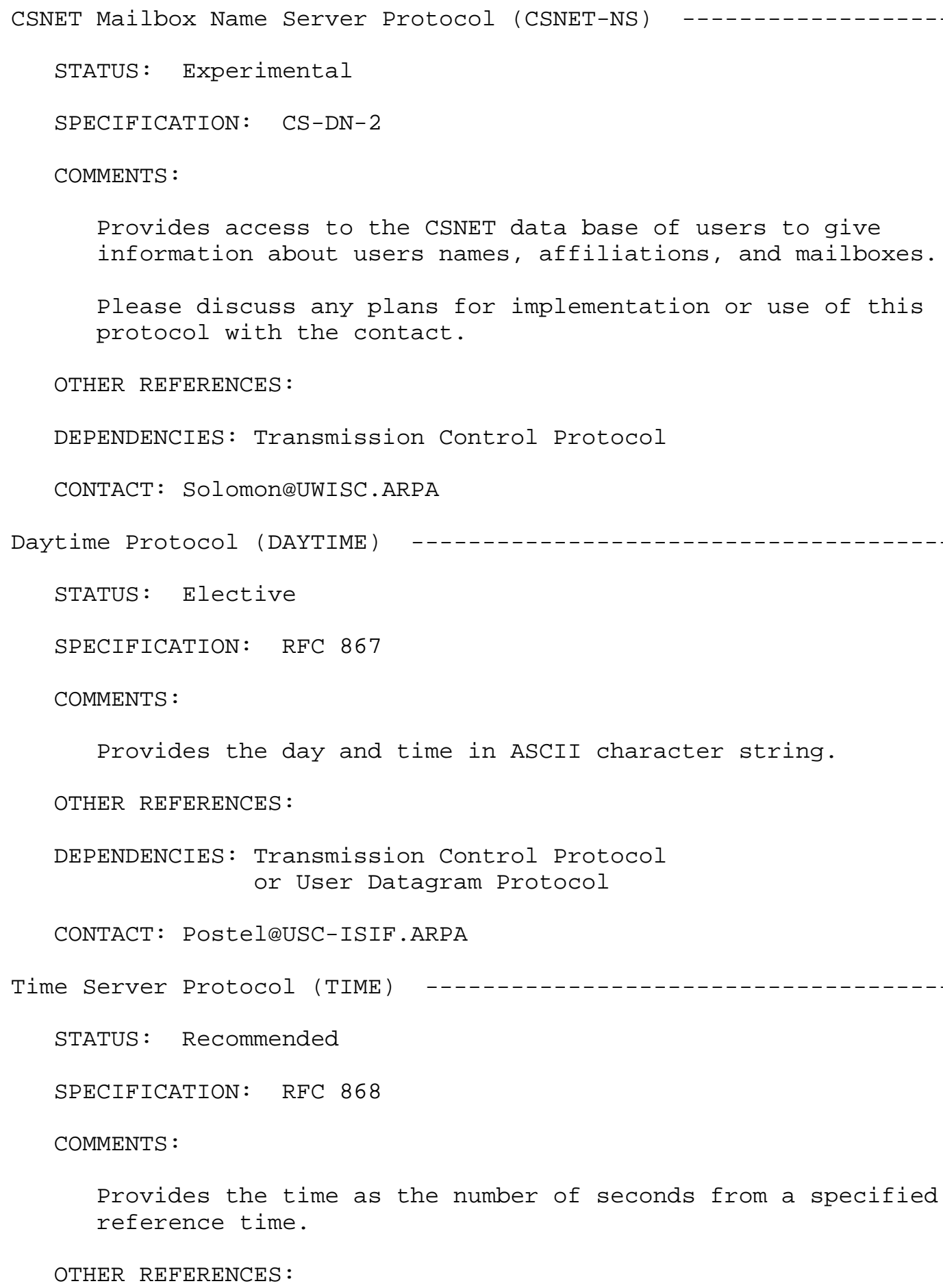




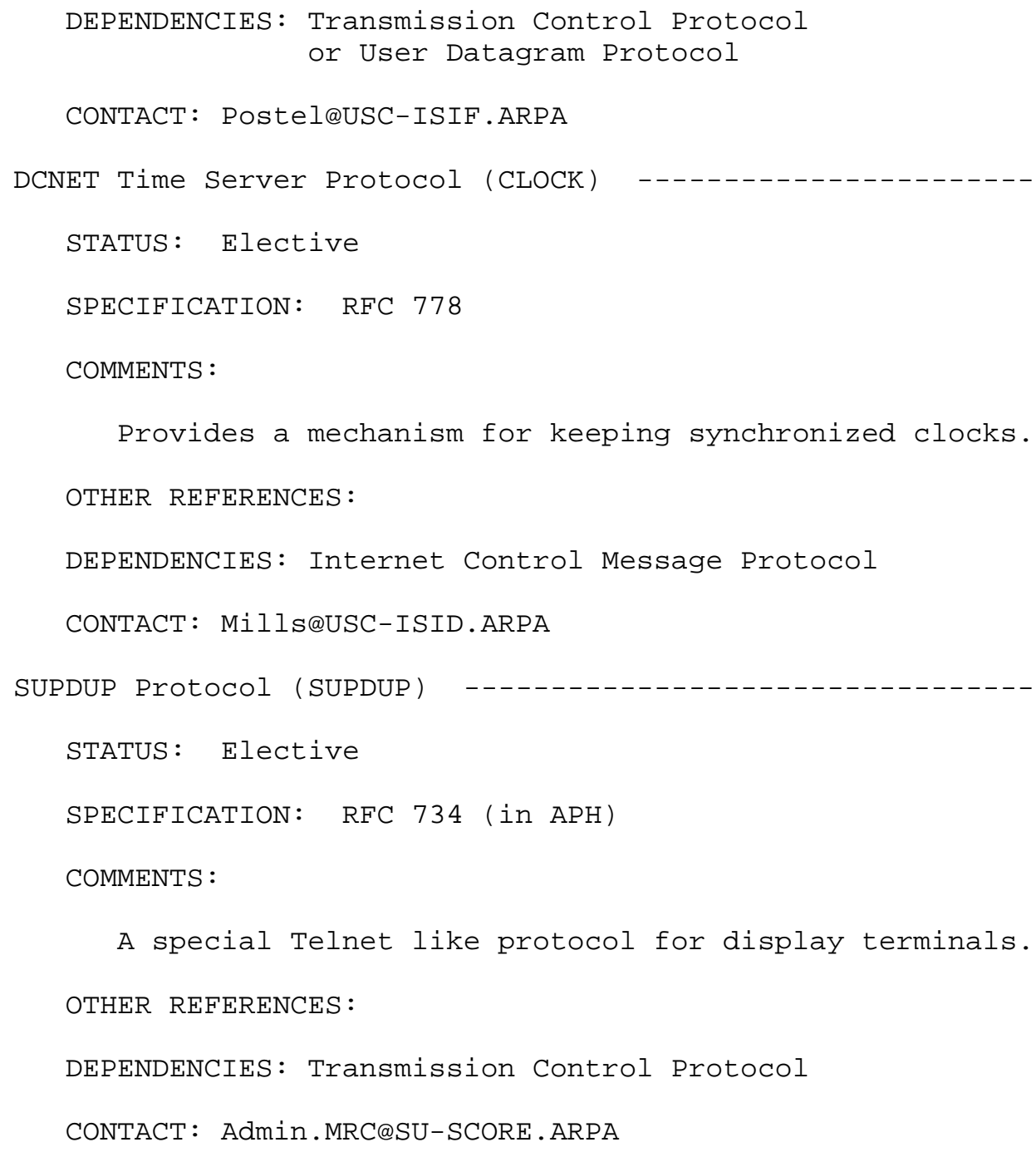




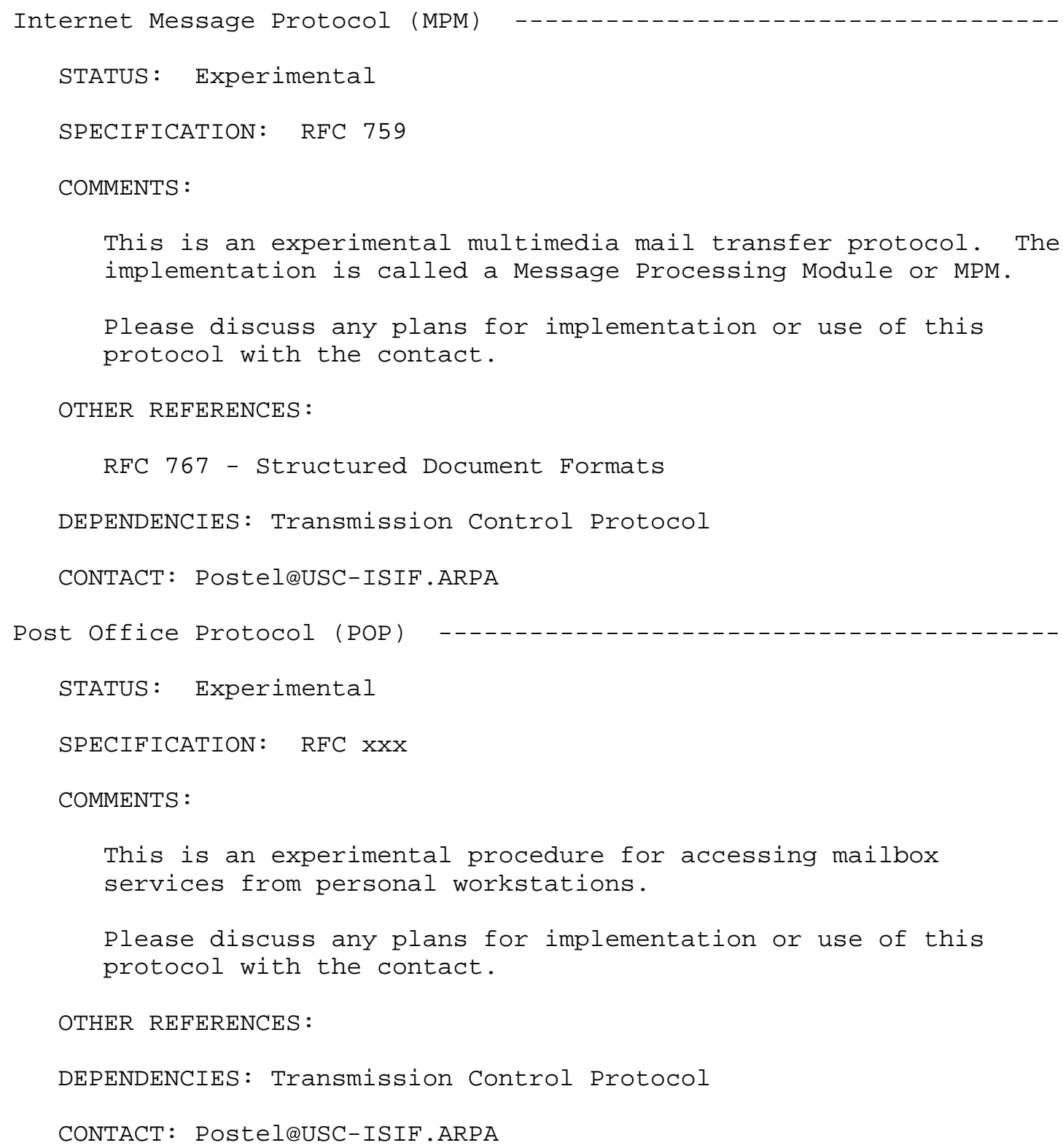


Network Standard Text Editor (NETED)

STATUS: Elective

SPECIFICATION: RFC 569

COMMENTS :

Describes a simple line editor which could be provided by every Internet host.

OTHER REFERENCES:

DEPENDENCIES :

CONTACT: Postel@USC-ISIF.ARPA

Appendices

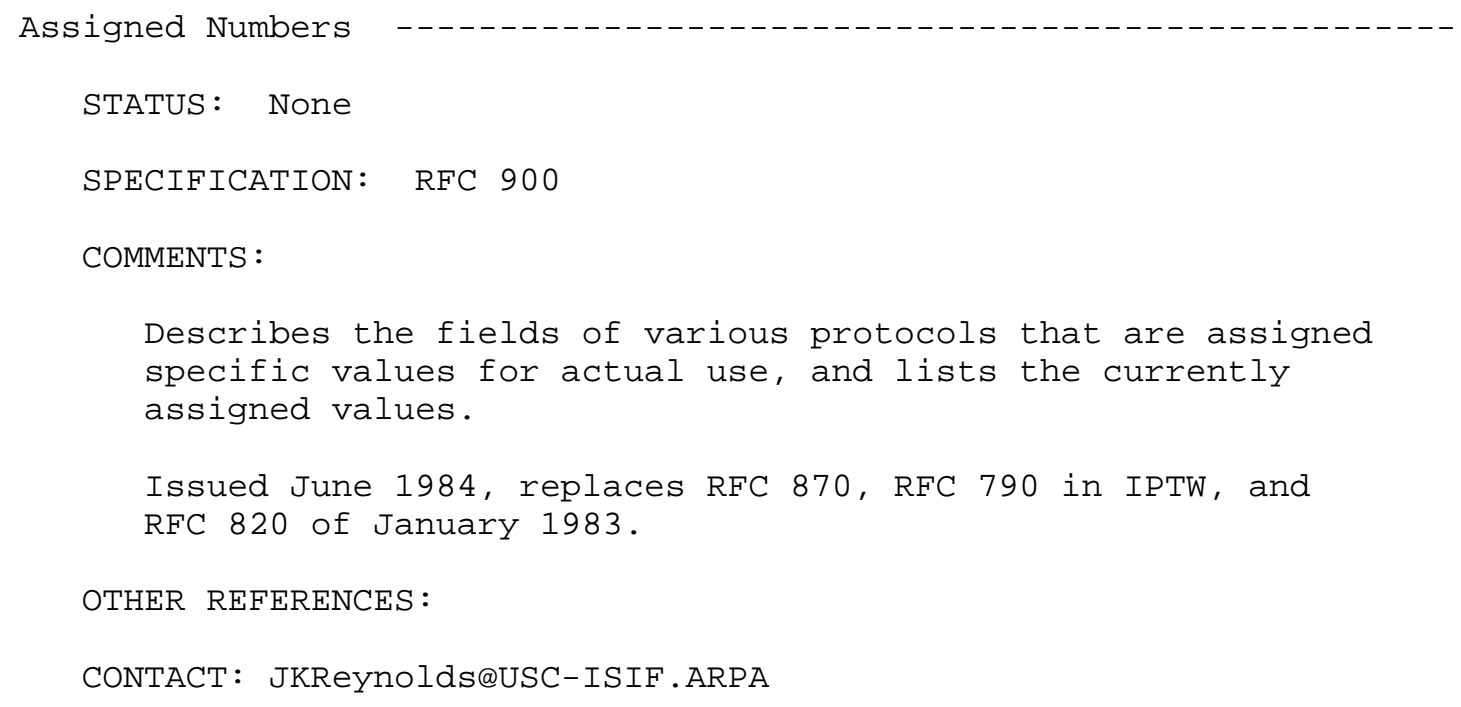




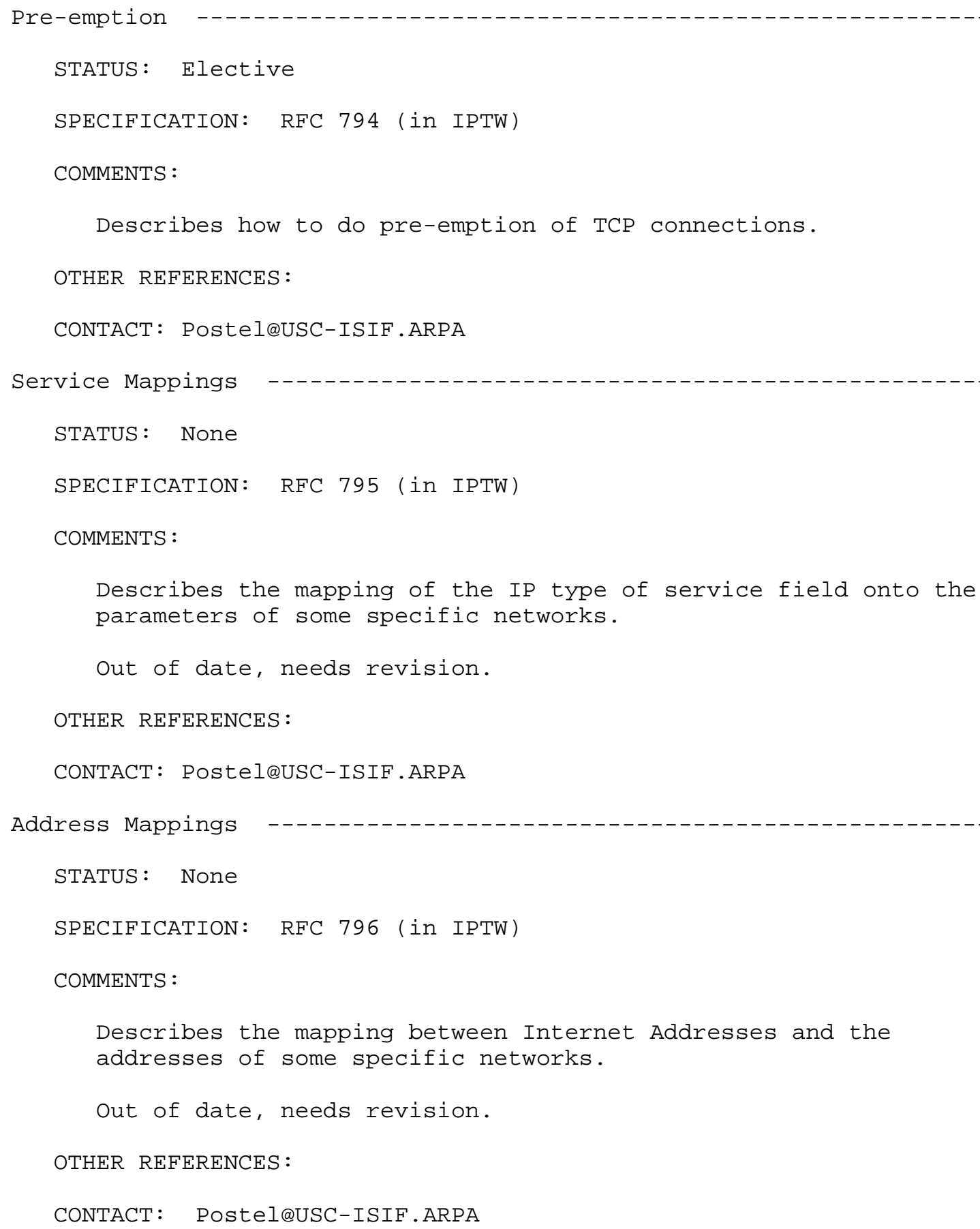




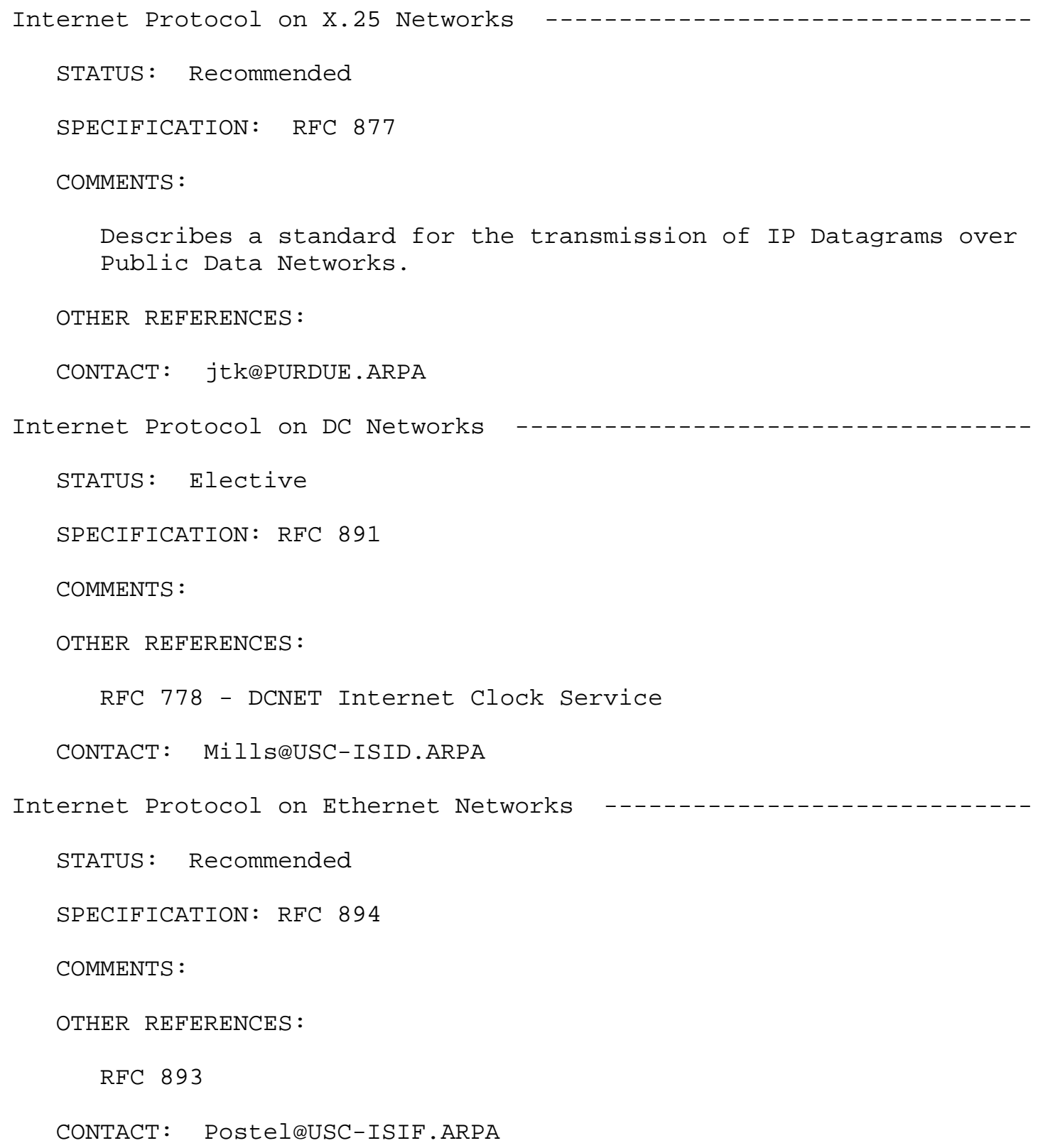




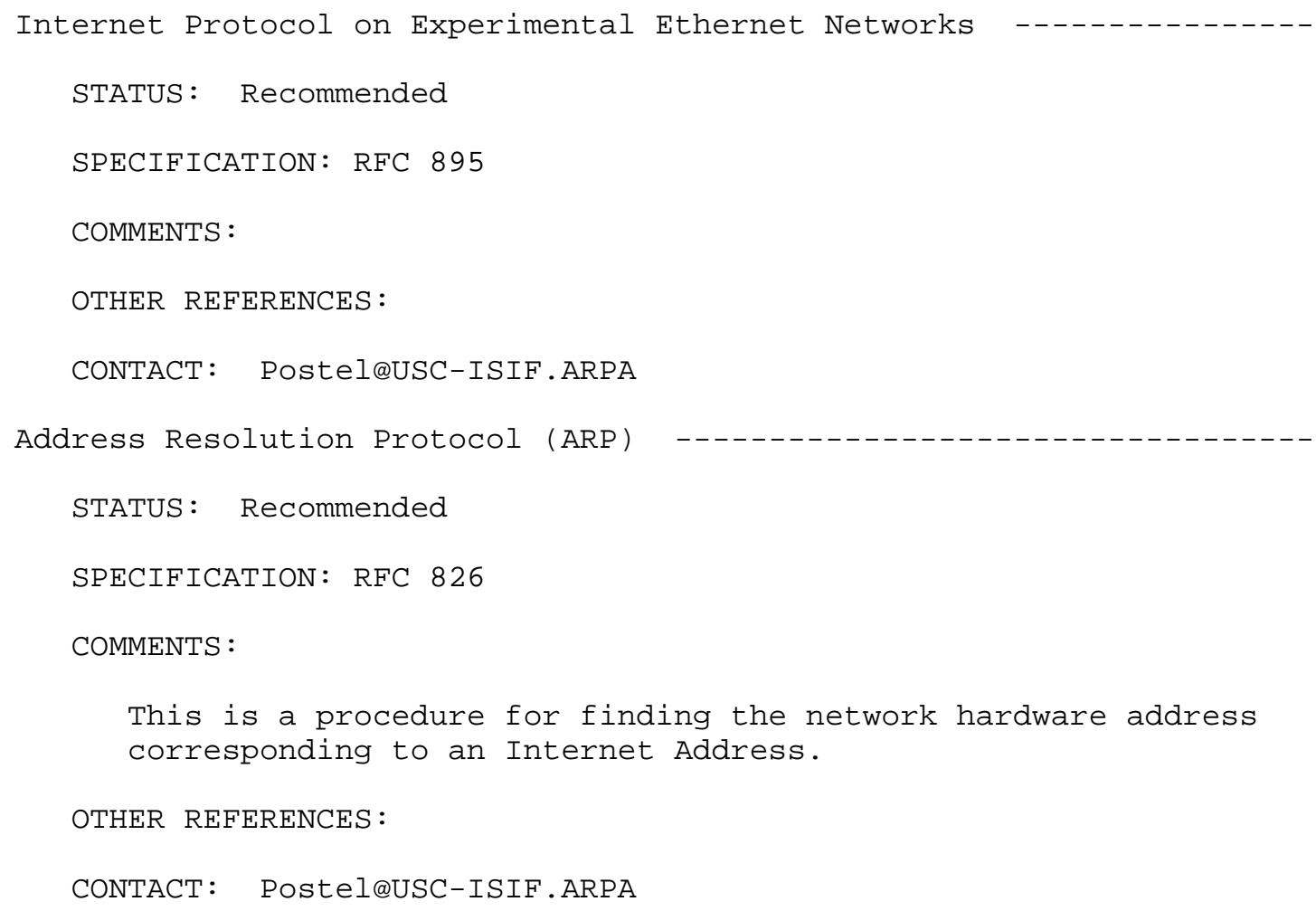

